\title{
DETERMINACIÓN DE VIDA ÚTIL EN ANAQUEL DE PAN LIBRE DE GLUTEN A BASE DE HARINA DE QUINUA (Chenopodium quinoa) ENVASADO EN POLIETILENO Y POLIPROPILENO
}

\author{
DETERMINATION OF SHELF LIFE OF GLUTEN- FREE BREAD MADE FROM \\ FLOUR OF QUINOA (Chenopodium quinoa) AND PACKED IN POLYETHYLENE \\ AND POLYPROPYLENE
}

\author{
${ }^{1}$ Gina Genoveva Toro Rodriguez; ${ }^{2}$ Thomas Ancco Vizcarra; ${ }^{2}$ David Juan Ramos Huallpartupa
}

\begin{abstract}
RESUMEN
En la investigación se determinó la vida en anaquel de pan libre de gluten a base de harina de quinua envasados en polietileno y polipropileno. Las muestras del pan libre de gluten se almacenaron 6 días bajo condiciones ambientales a $20^{\circ} \mathrm{C}$ y $89 \% \mathrm{HR}$; se efectuaron controles de acidez y recuento microbiológico según RM N ${ }^{\circ} 1020-2010$ / MINSA. Se determinó para el deterioro microbiológico, reacción de primer orden, $\mathrm{R}^{2}$ de 0,99 para polietileno y 0,93 para polipropileno. La vida útil en función al valor referencial de $1000 \mathrm{ufc} / \mathrm{g}$ del pan libre de gluten envasado en polipropileno es 3,27 días y en polietileno 4,13 días.
\end{abstract}

Palabras clave: Vida útil, pan libre de gluten, quinua.

ABSTRACT

The research determined the shelf life of breads of gluten- free bread made from flour of quinoa packed in polyethylene and polypropylene containers. The samples of gluten-free bread were stored 6 days under environmental conditions at $20^{\circ} \mathrm{Centigrades}$ and $89 \% \mathrm{HR}$, controls of acidity and microbiological counts according to RM N ${ }^{\circ}$ (Ministerial Resolution number) 1020 2010/MINSA (Ministry of Health) were made. Determining for the microbiological deterioration, a reaction of first order, with R2 of 0,99 for polyethylene and 0,93 for polypropylene. The useful life in function to the referential value of $1000 \mathrm{ufc} / \mathrm{g}$ of the gluten-free bread packaged in polypropylene is 3,27 days and in polyethylene 4,13 days.

Key words: useful Life, gluten-free bread, quinoa.

\section{INTRODUCCIÓN}

La vida útil de un alimento es el periodo de tiempo durante el cual mantiene una calidad adecuada, siempre que se garanticen las condiciones de conservación que se indican en el etiquetado, dependiendo tanto de las propias características de los alimentos como de las técnicas de conservación de los mismos; los estudios de vida útil aportan datos sobre cuánto tiempo un producto puede conservar inalteradas sus propiedades y mantener su calidad desde el momento en que el consumidor abre el envase. Los estudios de vida útil se basan en la teoría cinética, por la cual es posible determinar la velocidad de modificación de una propiedad o atributo del alimento. La mayoría de las reacciones de deterioro estudiadas en los alimentos se han caracterizado como de orden aparente 0 ó 1 , siendo catalogadas en primer orden: pérdida de ciertas vitaminas, desarrollo/muerte microbiana, pérdida de color por oxidación y pérdida de textura en tratamientos térmicos, se expresa mediante la ecuación siguiente: (Taoukis, Labuza y Saguy, 1997).

$$
V= \pm \frac{d A}{d t}=k\left(A_{0}\right)^{n}
$$

$\nu=$ velocidad de reacción

$A=$ atributo de calidad que estamos midiendo

$t=$ tiempo de vida comercial transcurrido

$k=$ constante de velocidad de reacción

$A_{0}=$ concentración inicial del atributo

$n=$ orden de la reacción

La demanda de alimentos sin gluten se encuentra en constante aumento, debido al incremento del número de consumidores que sufren de intolerancia al gluten trastorno conocido como enfermedad celíaca (Schober, 2009).

La enfermedad celíaca es uno de los trastornos más comunes en todo el mundo con una prevalencia estimada de $1 \%$ de la población en general (Catassi y Fasano, 2008).

La enfermedad celíaca consiste en una intolerancia permanente a la fracción de gliadina del trigo, a las prolaminas del centeno (secalinas), de la cebada (hordeínas) y posiblemente de la avena (avidinas) (Murray, 1999). Aunque la inclusión de avena en productos para pacientes con enfermedad celíaca es controvertida, ya que diversos estudios han demostrado que la ingesta en cantidades moderadas de la avena es bien tolerada por una gran mayoría de celiacos (Thompson, 2003).

\footnotetext{
Ingeniero Agroindustrial. Facultad de Ingenieria de la Universidad Nacional José María Arguedas. Andahuaylas-Perú.

${ }^{2}$ Master of Sciencie en Postcosecha y Marketing, Ingeniero Agroindustrial. Facultad de Ingenieria y Instituto de Investigación y Desarrollo Tecnológico de Recursos Andinos-IIDRA de la Universidad Nacional José Maria Arguedas. Andahuaylas-Perú.
} 
La reacción a la ingesta de gluten, por las víctimas de la enfermedad celíaca, es una inflamación del intestino delgado que conduce a la mala absorción de varios nutrientes importantes como hierro, ácido fólico, calcio y vitaminas liposolubles (Feighery, 1999; Farrell y Kelly, 2002). $\mathrm{El}$ único tratamiento que existe es mantener una dieta libre de gluten. La mayor parte de productos de panificación para celiacos, son elaborados a base de harina de arroz, debido a su sabor suave, color blanco, la digestibilidad de los hidratos de carbono y la hipoalergenicidad de sus proteínas (Rosell y Marco, 2008; Neumann y Bruemmer, 1997).

A pesar de las ventajas, anteriormente descritas, las proteínas del arroz son técnicamente inadecuadas para el procesamiento de alimentos, debido a que son extremadamente insolubles y muy hidrofóbicas (Lumdubwong y Seib, 2000). Estas características son responsables de que la harina de arroz sea incapaz de formar una red proteica cuando se mezcla con agua. Como consecuencia, el dióxido de carbono formado durante la fermentación no puede ser retenido en las masas de harina de arroz, originando productos con un bajo volumen específico y de migas duras (He y Hoseney, 1991).

Los granos andinos, como la kiwicha y otros afines cultivados en los Andes, son plantas que forman semillas ricas en almidón. Tienen excelentes propiedades nutritivas, ácidos grasos poliinsaturados y alto contenido en minerales. Según Alvarez-Jubete et al. (2009), las harinas de estos granos no contienen proteínas que formen el gluten, convirtiéndose en una alternativa atractiva como nuevo ingrediente en la elaboración de productos para celiacos, ya que la mayoría de los productos libres de gluten en el mercado son de muy mala calidad nutricional. La vida en anaquel es determinada para cada alimento en particular (Kennt et al., 1997) y el hecho de incorporar nuevos ingredientes en el diseño de nuevos producto, puede traer consigo variación de la vida de anaquel del alimento. El alimento es intrínsecamente perecedero $y$, en función de sus características físicas y químicas, de sus propiedades y las condiciones de almacenamiento, llegará un momento en que cualquiera de sus cualidades de calidad no serán aceptables o se convertirán en perjudiciales para el consumidor.

\section{MATERIALES Y MÉTODOS}

\section{Lugar de ejecución}

El trabajo de investigación se realizó en el Laboratorio de Análisis Físico-Químico de los Alimentos, Laboratorio de microbiología, Laboratorio de Investigación y en la Planta Piloto de Alimentos (TAPA) de la Facultad de Industrias Alimentarias de la Universidad Nacional Agraria La Molina.

\section{Materiales}

\section{- Materiaprima:}

Harina de quinua, variedad "blanca de Hualhuas", proveída por la empresa Insumos Mantaro ${ }^{\circledR}$.

\section{- Insumos:}

Almidón de papa UNO ${ }^{\circledR}$, Goma Xantano MONTANA $($, levadura instantánea FLEISCHMANN $($, aceite vegetal PRIMOR ${ }^{\circledR}$, azúcar POMALCA®, sal y agua de mesa SAN

\section{CARLOS $®$.}

- Materiales:

Moldes para pan, $10 \times 5 \times 5 \mathrm{~cm}$, bolsas plásticas de polietileno, polipropileno y laminada, espátulas de repostería, baguetas, beakers de $100 \mathrm{ml}$ y $250 \mathrm{ml}$, fiolas de $10 \mathrm{~mL}, 25 \mathrm{ml}$ y $50 \mathrm{ml}$, matraz de Erlenmeyer de $50 \mathrm{~mL}$ y 100 $\mathrm{ml}$, probeta de $250 \mathrm{~mL}$ y $500 \mathrm{ml}$, bureta bombilla de succión y termómetro

- Equipos:

Horno eléctrico, batidora, determinador de actividad de

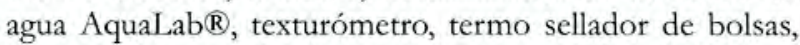
balanza de precisión $(0,1 \mathrm{~g})$, balanza analítica $(0,0001 \mathrm{~g})$, estufa, incubadora.

\section{Metodología de análisis}

\section{Elaboración del pan}

El diagrama de flujo de las operaciones a realizar, para la formulación óptima, se observa en la Figura 1.

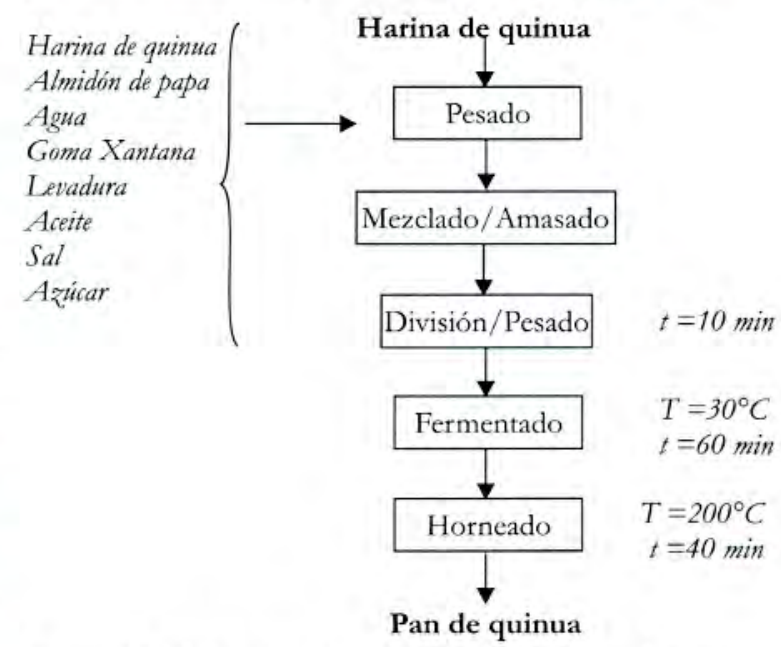

Figura $\mathrm{N}^{\circ} 1$. Elaboración de panes libres de gluten a partir de harina de quinua

\section{Controles}

Se realizaron controles de humedad, acidez (expresado en ácido sulfúrico) y microbiológico (mohos). Se almacenarán 5 muestras de pan por separado, en envases de polietileno y polipropileno a condiciones ambientales de $20^{\circ} \mathrm{C}$ y humedad relativa $89 \%$.

- Acidez: Se determinó por titulación. Método ISO 7305 (1986) - o - AOAC 939.05. Expresado en ácido sulfúrico

- Microbiológico: Se realizó recuento de mohos según RM $\mathrm{N}^{\circ}$ 1210-2010, en agar OGA (Oxitetraciclina-Glucosa Agar) con incubación a $22^{\circ} \mathrm{C}$ de 3 hasta 5 días.

\section{Análisis estadístico}

Los resultados fisicoquímicos en el producto terminado, fueron sometidos a prueba de comparación para determinar si existen diferencias significativas entre las muestras y el control (Gutiérrez y de la Vara, 2003) al 95\% de probabilidad.

\section{Determinación de vida útil}

Las muestras de pan de molde se dispusieron en envases de polietileno y polipropileno transparente $y$ fueron 
Toro, G. et al., Determinación de vida útil en anaquel de pan libre de gluten a base de harina de quinua (Chenopodium quinoa) envasado en polietileno y polipropileno.

almacenados a $21^{\circ} \mathrm{C}$ y $80 \% \mathrm{HR}$.

Se realizó muestreo a $1,2,3,4$ y 5 días los resultados físico-químicos fueron evaluadas por la cinética de reacción de deterioro, se determinó el orden de reacción y con el parámetro crítico se despejó la vida útil según la ecuación siguiente:

$$
\begin{aligned}
& \frac{d A}{d t}= \pm k A^{n} \\
& A=A_{0} * e^{ \pm k t}
\end{aligned}
$$

\section{RESULTADOS Y DISCUSIÓN}

\section{Análisis de muestras}

En las tablas 1 y 2 se puede observar los valores de recuento microbiológico de mohos y acidez total, tomados en los 5 días.

Tabla 1. Valores de recuento microbiológico de mohos

\begin{tabular}{ccc}
\hline Días & \multicolumn{2}{c}{ Tipo de Envase } \\
& Polipropileno & Polietileno \\
\hline 0 & $<10 \mathrm{ufc} / \mathrm{g}$ & $<10 \mathrm{ufc} / \mathrm{g}$ \\
1 & $30 \mathrm{ufc} / \mathrm{g}$ & $<10 \mathrm{ufc} / \mathrm{g}$ \\
2 & $40 \mathrm{ufc} / \mathrm{g}$ & $<10 \mathrm{ufc} / \mathrm{g}$ \\
3 & $1,9 \times 10^{\wedge} 2 \mathrm{ufc} / \mathrm{g}$ & $10 \mathrm{ufc} / \mathrm{g}$ \\
4 & $55 \times 10^{\wedge} 2 \mathrm{ufc} / \mathrm{g}$ & $3,5 \times 10^{\wedge} 2 \mathrm{ufc} / \mathrm{g}$ \\
5 & $54 \times 10^{\wedge} 3 \mathrm{ufc} / \mathrm{g}$ & $53 \times 10^{\wedge} 3 \mathrm{ufc} / \mathrm{g}$ \\
\hline
\end{tabular}

Realizando una comparación entre los dos tratamientos para el parámetro biológico, con una prueba de t obtenemos un valor $\mathrm{P}=0,93$, determinándose que no existe diferencia significativa entre grupos de estudio, los valores determinados y comparados con los establecidos en la RM N ${ }^{\circ} 1020-2010$ / MINSA, en ambos casos supera los valores de mohos y levaduras a los 5 días.

Tabla 2. Valores de acidez total (\% de ácido sulfúrico en base seca)

\begin{tabular}{ccc}
\hline Días & \multicolumn{2}{c}{ Tipo de Envase } \\
& Polipropileno & Polietileno \\
\hline 0 & 0,457 & 0,457 \\
1 & 0,338 & 0,355 \\
2 & 0,345 & 0,387 \\
3 & 0,348 & 0,372 \\
4 & 0,512 & 0,429 \\
5 & 0,353 & 0,338 \\
\hline
\end{tabular}

Realizando una comparación entre ambos tratamientos para el parámetro de acidez total, con una prueba de t obtenemos un valor- $\mathrm{P}=0,95$, el cual nos indica que no existe diferencia significativa entre ambos grupos. La acidez para el pan libre de gluten envasado en polipropileno a los 04 días supera los parámetros establecidos en la $\mathrm{RM} \mathrm{N}^{\circ}$ 1020-2010/ MINSA, a diferencia del pan envasado en polietileno.

\section{Cálculo de vida útil}

El cálculo de vida útil se realizó en función al conteo microbiológico. En la figura 2 observamos el orden

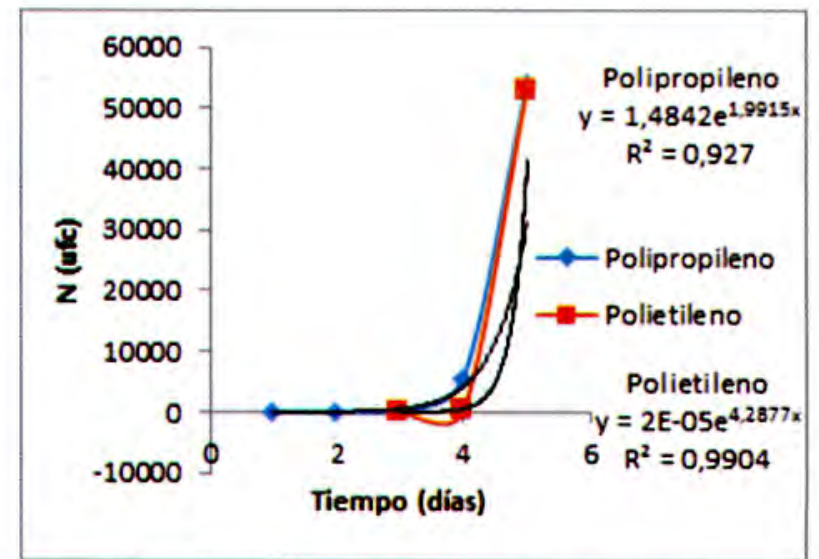

Figura $\mathrm{N}^{\circ} 2$. Unidades formadoras de colonia respecto a días

de la reacción de deterioro.

Podemos observar que ambas curvas se ajustan más a una cinética de reacción de orden uno, siendo la más notoria el envasado en polietileno ya que alcanza un $\mathrm{R}^{2}=$ 0,99 .

En la figura 3 se muestra las tendencias de crecimiento de microorganismos.

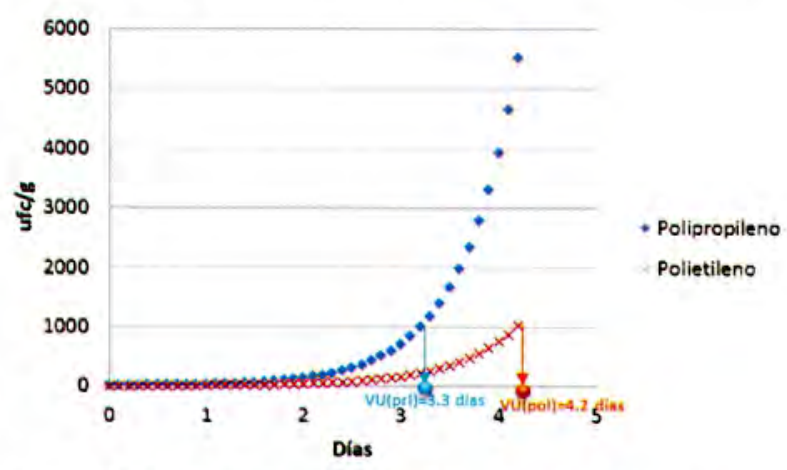

Figura $N^{\circ} 3$. Crecimiento microbiano según envase

Las ecuaciones para ambas curvas son del tipo exponencial y son las siguientes:

$$
\begin{aligned}
& N_{\text {polietileno }}=0,00005 e^{4,2877 t} \\
& N_{\text {polipropileno }}=1,4842 e^{1,9915 t}
\end{aligned}
$$

Donde "N" es la población microbiana predicha y " $t$ " es el tiempo en días.

Conociendo que el valor mínimo permisible es 1000 ufc/g (según RM No 1020-2010/ MINSA) se estimó la vida útil la cual se muestra en la Tabla 3.

Tabla 3. Valores de vida útil calculada según el tipo de envase

\begin{tabular}{cc}
\multicolumn{2}{c}{ Vida útil (dias) } \\
Polipropileno & Polietileno \\
\hline 3,27 & 4,13 \\
\hline
\end{tabular}

Como se observa el envasado en polietileno nos da una mayor vida útil, aunque se debe tener cuidado con esta predicción ya que el valor de ajuste que se encontró no era lo suficiente para afirmar categóricamente. 
Toro, G. et al., Determinación de vida útil en anaquel de pan libre de gluten a base de harina de quinua (Chenopodium quinoa) envasado en polietileno y polipropileno.

\section{CONCLUSIÓN}

La vida útil del pan libre de gluten a base de harina de quinua es mayor en envase de polietileno estimado a un orden de reacción de tipo uno.

\section{REFERENCIAS BIBLIOGRÁFICAS}

Alvarez-Jubete, L.; Arendt, E.; Gallagher, E. (2009). Nutritive value and chemical composition of pseudocereals as gluten-free ingredients. International Journal of Food Science and Nutrition, 60(1) (Suppl. 4), $240-257$.

AOAC, (2002). Official Methods of Analysis of the Association of Official Analytical Chemists.

Catassi, C.; Fasano, A. (2008). Celiac disease. In E. K. Arendt, \& F. Dal Bello (Eds.), Gluten-free cereal products and beverages. London: Academic Press.

Farrell, J.; Kelly, P. (2002). Coeliac sprue. New Engl J Med 346(3):180-8.

Gutiérrez H. y De la Vara R.2003. Análisis y Diseño de experimentos. Ed. Mc Graw Hill Interamericana. México. $571 \mathrm{pp}$.

He, H.; Hoseney, R. (1991). Gas retention of different cereal flours. Cereal Chemistry, 68:334-

Kennet, J, V., Rotsteine, E., and Sing, P. R. (1997). Handbook of food engineering. Practice. CRC Press, New York

Lumdubwong, N.; Seib P. (2000). Rice starch isolation by alkaline protease digestion of wet-milled rice flour. Journal of Cereal Science, 200:63-74.

Murray, J. (1999). The widening spectrum of celiac disease. Am J Clin Nutr 69:354-65.

Neumann, H.; Bruemmer, J. (1997). Investigations with the production of gluten free bread and roll specialities. Getreide Mebl Brot 51, 50-55.

Rosell, C.; Marco, C. (2008). Rice. In: Arendt EK, Dal Bello F. (eds.) Gluten-free cereal products and beverages. Elsevier Academic Press, Massachusetts.

Schober, T. (2009). Manufacture of gluten-free specialty breads and confectionery products. In E. Gallagher (Ed.), gluten-free food science and technology (pp. $130-180$ ). London: John

Taoukis, P.S.; Labuza, T. P.; Saguy, I. (1997). Kinetics of food deterioration and shelf-life prediction. En K. J. Valentas, E. Rotstein, R. P. Singh, (eds.) The Handbook Of Food Engineering Practice, Ch. 9, CRC Press, N.Y., EEUU, 363-405.

Thompson, T. (2003). Oats and the gluten-free diet. J Am Diet Assoc 103(3):376-379

Thompson, T. (2009). The nutritional quality of gluten-free foods. In E. Gallagher (Ed.), Gluten-free food science and technology. Oxford: Wiley-Blackwell

\section{Correspondencia:}

Gina Toro Rodriguez: gtoro@unajma.edu.pe

Thomas Ancco Vizcarra: thoanviz@gmail.com

David Ramos Huallpartupa: davisrh@gmail.com
Fecha de Recepción: 7/11/2014

Fecha de Aceptación: 10/12/2014 